\title{
Progress in neutron reflectometry instrumentation
}

\author{
Giovanna Fragneto ${ }^{1, a}$ and Alain Menelle $^{2}$ \\ 1 Institut Laue-Langevin - 6 rue Jules Horowitz, BP 156, 38042 Grenoble, France \\ ${ }^{2}$ Laboratoire Léon Brillouin, CEA-CNRS UMR 12 - 91191 Gif-sur-Yvette, Saclay, France
}

Received: 11 October 2011

Published online: 11 November 2011 - (C) Società Italiana di Fisica / Springer-Verlag 2011

The last three decades have seen a flourishing of neutron reflectometry (NR) techniques for the study of layers at interfaces and they are today recognized as powerful tools for the study of a variety of systems ranging from surface chemistry (surfactants, polymers, lipids, proteins and mixtures adsorbed at liquid/fluid and solid/fluid interfaces), to surface magnetism (ultrathin Fe films, magnetic multilayers, superconductors) and solid films (Langmuir-Blodgett films, thin solid films, multilayers, polymer films). For studies at interfaces, neutron reflection offers many advantages with respect to traditional techniques as well as to X-ray reflection. In particular, because of the short wavelengths available, it has a resolution of a fraction of a nanometer, it is non-destructive and can be applied to buried interfaces which are not easily accessible to other techniques. In addition, it provides the advantage that isotopic substitution can be used to achieve large contrasts in the scattering length density. Lateral inhomogeneities cannot be probed by specular reflection and off-specular studies become necessary.

When a neutron beam arrives on a planar surface, at a grazing angle, the atoms of the surface act as a mirror and, as for light, the beam is nearly totally reflected. When a thin layer is deposited on such surface, there will be interference between the beam reflected by the surface and the beam reflected by the interface. The technique is very sensitive to the thickness of the layer, to the composition as well as to the roughness of the interfaces. Its distinctive value is that deuterium substitution may be used either to enhance the sensitivity to the structure of a particular component of the surface layer or to resolve the ambiguities resulting from the loss of phase information that is characteristic of all scattering experiments. The former feature often allows the successful study of a system by NR but not by X-ray reflection (XR) and the latter feature often gives NR an effective resolution that eclipses the intrinsically superior resolution of XR. Isotopic labelling also allows NR to give the composition of the layer independently of its structure. Even thirty years after its first implementation [1], NR remains the only technique that gives composition and structure from a liquid surface. Structure is so strongly linked to composition that any structural determination is almost useless without knowledge of the composition [2].

Specular reflectivity, defined as the ratio between the reflected and the incoming intensities of a neutron beam, is measured as a function of the wave vector transfer, $Q=4 \pi / \lambda \sin \theta$ (where $\lambda$ is the wavelength and $\theta$ the angle of the incoming beam to the surface). Measurements can be done either by using a fixed wavelength and performing $\theta-2 \theta$ scans (monochromatic mode) - the resolution in such measurements is determined by both angular and wavelength spread - or by using a white neutron beam at a fixed angle (time-of-flight mode, TOF); the values of $Q$ are determined from the time of arrival of each neutron as a function of its wavelength.

For liquid samples the incident angle cannot be varied by tilting the sample, as is usually done with solid substrates. It is necessary to use an inclined beam and therefore to translate vertically the sample to make sure that the inclined beam hits the center every time the inclination changes. In the monochromatic mode the incident angle is varied for each point of the reflectivity profile and therefore the sample height must be adjusted each time.

Advantages of the TOF over the monochromatic mode include:

- fewer movements of the sample (normally 2 incident angles are used);

- the possibility to measure a wide $Q$ range in kinetics measurements;

- the possibility to vary the resolution with a chopper system.

The number of reflectometers around the world is steadily increasing. For thin-film studies (few nm thick) instruments with high flux able to determine structures in the nanometer range are best suited and remarkable results have been obtained on instruments like NG1 or AND/R at NIST [3], D17 at the ILL [4] and SURF at ISIS [5].

\footnotetext{
a e-mail: fragneto@ill.eu
} 
Enormous progress has been achieved in the last few years both on instrumentation and sample environment and data analysis. The quest for high-flux instruments allowing the improvement of the accessible resolution as well as the possibility of performing kinetics studies has brought to the design and building of new reflectometers in most neutron facilities around the world and the upgrade of old ones. The increasing popularity of the technique is such that reflectometers were the first instruments to be built in new neutron sources like ANSTO (Sidney, Australia), J-PARC (Tokai, Japan), SNS (Oak Ridge, USA), FRM-II (Munich, Germany) and ISIS-TS2 (Didcot, UK). The time-of-flight technique is the technique of choice for all new reflectometers with the notable exception of MAGIK at NIST (USA), a reflectometer that is currently being built and that aims at building up quickly a picture of the scattering crosssection (i.e. measures off-specular reflectivity) by measuring a number of scattered-beam angle simultaneously (with a position-sensitive detector).

This focus point is a collection of papers covering both the upgrade of old reflectometers (see contributions on EROS at LLB by F. Cousin et al. and SPEAR at LANL by M. Dubey et al.), the design and performance of recently commissioned ones (FIGARO at ILL by R.A. Campbell et al., INTER, POLREF and OFFSPEC at ISIS-TS2 by J.R.P. Webster et al., SOFIA at J-PARC/MLF by N.L. Yamada et al.) as well as two methodological contributions useful for future developments of the technique like the possibility to measure inelastic scattering on a neutron reflectometer (by A. Wildes) and to do real measurements involving prism refraction to deduce the wavelength in place of choppers, opening up the possibility of sub-second kinetics studies (by R. Cubitt et al.).

Table 1 below points out at some recently commissioned (in the last five years) time-of-flight reflectometers, see also $[6]$ :

Table 1. Some time-of-flight reflectometers recently commissioned.

\begin{tabular}{|c|c|c|c|}
\hline Instrument & $\begin{array}{l}\text { Scattering } \\
\text { plane }\end{array}$ & Options & Technical paper \\
\hline $\begin{array}{l}\text { REFSANS } \\
\text { (FRM-II) }\end{array}$ & Vertical & Grazing incidence small angle neutron scattering. & {$[7]$} \\
\hline $\begin{array}{l}\text { Platypus } \\
\text { (ANSTO) }\end{array}$ & Vertical & Specular and off-specular scattering. Polarised neutrons. & {$[8]$} \\
\hline $\begin{array}{l}\text { FIGARO } \\
\text { (ILL) }\end{array}$ & Vertical & $\begin{array}{l}\text { Simultaneous use of a Brewster Angle Microscope (BAM) } \\
\text { during measurements and the possibility to strike the interface } \\
\text { from above and below in a wide } q \text { range. }\end{array}$ & This issue \\
\hline $\begin{array}{l}\text { BioRef } \\
(\mathrm{HZB})\end{array}$ & Horizontal & Sample environment for in situ infrared spectroscopy. & {$[9]$} \\
\hline $\begin{array}{l}\text { INTER } \\
(\text { ISIS-TS2) }\end{array}$ & Vertical & Small sample sizes. & This issue \\
\hline $\begin{array}{l}\text { POLREF } \\
\text { (ISIS-TS2) }\end{array}$ & Vertical & Polarisation and Analysing systems. & This issue \\
\hline $\begin{array}{l}\text { OFFSPEC } \\
\text { (ISIS-TS2) }\end{array}$ & Vertical & $\begin{array}{l}\text { Neutron spin echo analysis system that will simultaneously } \\
\text { provide data on in plane structures from } 5 \text { to } 200 \mathrm{~nm} \text {. } \\
\text { Polarised neutrons. }\end{array}$ & This issue \\
\hline $\begin{array}{l}\text { SOFIA } \\
\text { Reflectometer } \\
(\text { J-PARC })\end{array}$ & Vertical & $\begin{array}{l}\text { Specular, off specular, near-surface small angle scattering. } \\
\text { Polarised neutrons. }\end{array}$ & This issue \\
\hline $\begin{array}{l}\text { Liquids } \\
\text { Reflectometer } \\
\text { (SNS) }\end{array}$ & Vertical & $\begin{array}{l}\text { Specular, off specular, near-surface small angle scattering, and } \\
\text { crystalline diffraction geometries. }\end{array}$ & n.a. \\
\hline
\end{tabular}

The examples reported in this paper collection provide an indication of the healthy status of this technique that has struggled to find its deserved first-class position in the realm of neutron scattering methods and allow to foresee an exciting future both for the science that is being made possible and for technical progress. 


\section{References}

1. J.B. Hayter, R.R. Highfield, B.J. Pullman, R.K. Thomas, A.I. McMullen, J. Penfold, J. Chem. Soc., Faraday Trans. I 77, 1437 (1981).

2. J. Penfold, R.C. Ward, W.G. Williams, J. Phys. E 20, 1411 (1987).

3. J.A. Dura, D.J. Pierce, C.F. Majkrzak, N.C. Maliszewskyj, D.J. McGillivray, M. Lösche, K.V. O’Donovan, M. Michailescu, U. Perez-Salas, D.L. Worcester, S.H. White, Rev. Sci. Instrum. 77, 074301 (2006).

4. R. Cubitt, G. Fragneto, Appl. Phys. A: Mater. Sci. Process 74, S329 (2002).

5. J. Penfold, R.M. Richardson, A. Zarbakhsh, J.R.P. Webster, D.G. Bucknall, A.R. Rennie, R.A.L. Jones, T. Cosgrove, R.K. Thomas, J.S. Higgins, P.D.I. Fletcher, E. Dickinson, S.J. Roser, I.A. McLure, A.R. Hillman, R.W. Richards, E.J. Staples, A.N. Burgess, E.A. Simister, J.W. White, J. Chem. Soc., Faraday Trans. 93, 3899 (1997).

6. S. Teixeira et al., Chem. Phys. 345, 133 (2008).

7. R. Kampmann, M. Haese-Seiller, V. Kudryashov, B. Nickel, C. Daniel, W. Fenzl, A. Shreyer, E. Sackmann, J. Rädler, Physica B 385-386, 1161 (2006).

8. M. James, A. Nelson, S.A. Holt, T. Saerbeck, W.A. Hamilton, F. Klose, Nucl. Instrum. Methods Phys. Res. A 632, 112 (2011).

9. M. Strobl, R. Steitz, M. Kreuzer, M. Rose, H. Herrlich, F. Mezei, M. Grunze, R. Dahint, Rev. Sci. Instrum. 82, 055101 (2011). 